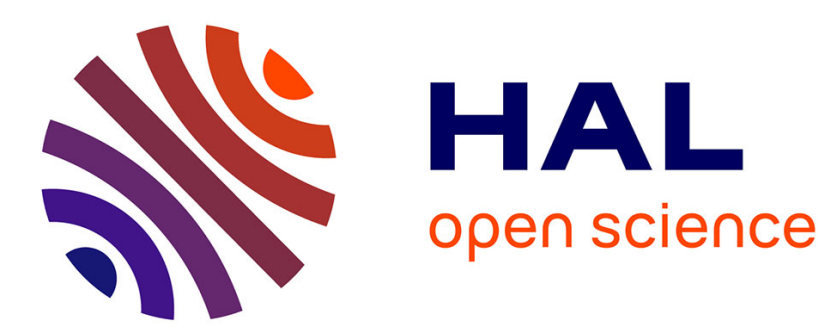

\title{
Stabilization of a magnetic island by localized heating in a tokamak with stiff temperature profile
}

\author{
Patrick Maget, Fabien Widmer, Olivier Février, Xavier Garbet, Hinrich
}

Lütjens

\section{- To cite this version:}

Patrick Maget, Fabien Widmer, Olivier Février, Xavier Garbet, Hinrich Lütjens. Stabilization of a magnetic island by localized heating in a tokamak with stiff temperature profile. Physics of Plasmas, 2018, 25, pp.022514. 10.1063/1.5021759 . hal-01614656

\author{
HAL Id: hal-01614656 \\ https://hal.science/hal-01614656
}

Submitted on 11 Oct 2017

HAL is a multi-disciplinary open access archive for the deposit and dissemination of scientific research documents, whether they are published or not. The documents may come from teaching and research institutions in France or abroad, or from public or private research centers.
L'archive ouverte pluridisciplinaire HAL, est destinée au dépôt et à la diffusion de documents scientifiques de niveau recherche, publiés ou non, émanant des établissements d'enseignement et de recherche français ou étrangers, des laboratoires publics ou privés. 


\title{
Stabilization of a magnetic island by localized heating in a tokamak with stiff temperature profile
}

\author{
Patrick Maget, Fabien Widmer, Olivier Février ${ }^{1}$, Xavier Garbet and Hinrich Lütjens ${ }^{1}$ \\ CEA, IRFM, F-13108 Saint Paul-lez-Durance, France. \\ ${ }^{1}$ Centre de Physique Théorique, Ecole Polytechnique, CNRS, France. \\ patrick.maget@cea.fr
}

\begin{abstract}
In tokamaks plasmas, turbulent transport is triggered above a threshold in the temperature gradient and leads to stiff profiles. This particularity, neglected so far in the problem of magnetic island stabilization by a localized heat source, is investigated analytically in the present paper. We show that the efficiency of the stabilization is larger than previously estimated due to the reduced turbulence level inside the island.
\end{abstract}

The production of fusion energy in tokamaks requires the confinement of a hot plasma medium in nested magnetic surfaces. Magnetic islands can be damped using the injection of a localized current drive at their O-point, or by a localized heating Kurita et al. (1994); Hegna and Callen (1997); Lazzari and Westerhof (2009). In this later case, the stabilizing effect is provided by the reduction of the local plasma resistivity that depends on the temperature change produced by the local heating, and therefore on the properties of the heat transport that is mainly originating from turbulent processes. Theory Rebut et al. (1988); Dimits et al. (2000); Garbet et al. (2004) and experiments Imbeaux et al. (2001) show that turbulent transport is triggered above a critical temperature gradient, and leads to resilient (also refered to as stiff) profiles above this threshold, with a stiffness that is expected to be large in ITER Kinsey et al. (2011). Inside magnetic islands, where the temperature profile is flattened, a reduced diffusivity is expected Hornsby et al. (2011) and effectively measured Inagaki et al. (2004); Ida et al. (2012). The consequences of this kind of transport rule has recently been investigated for nonlinear island saturation Fitzpatrick (2017). Here we show analytically that profile stiffness leads to much more efficient stabilization process by localized heating than anticipated when considering a uniform diffusivity.

\section{Stiffness model}

We adopt a simple model for the heat diffusivity, that incorporates plasma stiffness in the vicinity of a reference state where turbulent transport equilibrates the incoming heat flux:

$$
\chi_{\perp}=\chi_{\perp}^{0}\left|T^{\prime} / T_{e q}^{\prime}\right|^{\sigma-1}
$$

where $T$ is the temperature and the prime refers to the derivative relative to the radial co-ordinate, $\sigma$ is the stiffness, the "eq" subscript refers to the equilibrium situation without magnetic island and without additional heating from RF waves, and $\chi_{\perp}^{0}$ is the heat diffusivity in this reference case. In this representation, anomalous transport starts growing above a critical gradient $\left|T_{\text {crit }}^{\prime} / T_{\text {eq }}^{\prime}\right|=1-1 / \sigma$, with a soft transition between sub- and over-critical regimes. This formulation is consistent with the definition of stiffness given in Kinsey et al. (2011). The effect of local heating on island stabilization has only been addressed in the case $\sigma=1$ in previous works.

The energy balance equilibrates the heat flux with the volumic heat source $H$ :

$$
\nabla \cdot\left(N \chi_{\perp} \nabla T\right)=-H
$$

with $N$ the plasma density. Without island, after integrating on the plasma volume inside a radial position $r$ we obtain

$$
-T^{\prime}(r)=\frac{\int_{0}^{r} d r^{\prime} \mathcal{J}\left(r^{\prime}\right) H\left(r^{\prime}\right)}{\mathcal{J}(r) N(r) \chi_{\perp}(r)}=\frac{P(r)}{(2 \pi)^{2} \mathcal{J}(r) N(r) \chi_{\perp}(r)}
$$

\footnotetext{
${ }^{1}$ Now at Swiss Plasma Center, Ecole Polytechnique Fédérale de Lausanne, CH-1015 Lausanne, Switzerland.
} 
with $P(r)$ the power injected inside $r$ and $\mathcal{J}$ the Jacobian of the co-ordinate system $(r, \theta, \varphi)$, with $\theta$ and $\varphi$ the poloidal and toroidal angles, that in the large aspect ratio limit is $\mathcal{J} \approx r R$ with $R$ the major radius of the torus. At equilibrium, $H=H_{e q}$ and

$$
-T_{e q}^{\prime}(r)=\frac{\int_{0}^{r} d r^{\prime} \mathcal{J}\left(r^{\prime}\right) H_{e q}\left(r^{\prime}\right)}{\mathcal{J}(r) N(r) \chi_{\perp}^{0}(r)}=\frac{P_{e q}(r)}{(2 \pi)^{2} \mathcal{J}(r) N(r) \chi_{\perp}^{0}(r)}
$$

When the plasma is heated by RF waves, $H=H_{e q}+H_{R F}, P=P_{e q}+P_{R F}$ and

$$
-T^{\prime}(r)=\frac{P_{e q}(r)}{(2 \pi)^{2} \mathcal{J}(r) N(r) \chi_{\perp}^{0}(r)}\left(1+\frac{P_{R F}}{P_{e q}}\right)^{1 / \sigma}
$$

The gradient increase due to the RF heating is reduced as expected when the stiffness is large.

\section{Heating at the O-point of an island}

We now consider the magnetic equilibrium of a tokamak with major radius $R$ and minor radius $a$, a toroidal magnetic field $B_{z}$, a safety factor $q$ and magnetic shear $s=(r / q) d q / d r$ in the large aspect ratio limit. The magnetic perturbation with poloidal and toroidal mode numbers $m$ and $n$ associated with the magnetic island localized at $r=r_{s}$ where $q=m / n$ is $\tilde{B}=\nabla \times \tilde{\psi} \mathbf{e}_{\mathbf{z}}$. The magnetic flux surfaces are labelled by $\Omega$ with $\Omega=8(x / w)^{2}-\cos (m \alpha)$ with $x=r-r_{s}, \alpha=\theta-(n / m) \varphi$, and $w$ the island total width. We have the relation

$$
\frac{\tilde{\psi}}{B_{z}}=-\frac{w^{2}}{16} \frac{n s}{R m} \cos (m \alpha)
$$

In the following we use the notations $g(\alpha) \equiv \cos (m \alpha)$ and $\psi_{1} \equiv \frac{w^{2}}{16} \frac{B_{z} n s}{R m}$. After integrating equation 2 in the interval $\left(2 \sqrt{2} x_{ \pm} / w\right)= \pm \Omega$, we obtain:

$$
\left(-\frac{d T}{d \Omega}\right)=\frac{\left(P_{e q} /(2 \pi)^{2}\right)^{1-1 / \sigma}}{N \chi_{\perp}^{0} \mathcal{J}_{s}}\left(\frac{w}{4 \sqrt{2}}\right)^{1+1 / \sigma}\left\{\frac{\int_{-1}^{\Omega} d \Omega^{\prime} \mathcal{J} \oint d \alpha \frac{H\left(\Omega^{\prime}, \alpha\right)}{\sqrt{\Omega^{\prime}+g}}}{\oint d \alpha(\Omega+g)^{\sigma / 2}}\right\}^{1 / \sigma}
$$

with $\mathcal{J}_{s}$ the Jacobian at the resonant surface. We assume that there is no other heat source in the island than RF power, with a constant value of $H_{R F}$ in the region $\Omega \in\left[-1, \Omega_{c}\right]$ or $\mu=(\Omega+1) / 2 \in\left[0, \mu_{c}\right]$, with $\mu_{c}<1$. This mimics a perfect O-point heating, and leads to

$$
\begin{aligned}
-\frac{d T}{d \Omega} & =\frac{P_{e q}^{1-1 / \sigma} P_{R F}^{1 / \sigma}(\Omega)}{(2 \pi)^{2} N \chi_{\perp}^{0} \mathcal{J}_{s}} \frac{w}{4 \sqrt{2}}\left\{\frac{\pi}{I_{\sigma}(\Omega)}\right\}^{1 / \sigma} \\
& =\left(-T_{s}^{\prime}\right)\left(\frac{P_{R F}^{t o t}}{P_{e q}}\right)^{1 / \sigma} \frac{w}{4 \sqrt{2}}\left\{\frac{\pi}{I_{\sigma}(\Omega)} \frac{E(\mu)+(\mu-1) K(\mu)}{E\left(\mu_{c}\right)+\left(\mu_{c}-1\right) K\left(\mu_{c}\right)}\right\}^{1 / \sigma}
\end{aligned}
$$

where $T_{s}^{\prime}=T_{e q}^{\prime}\left(r_{s}\right)$ and

$$
\begin{aligned}
I_{\sigma}(\Omega) & =\oint d \alpha(\Omega+g(\alpha))^{\sigma / 2} \\
& =2^{2+\sigma / 2} \mu^{1 / 2+\sigma / 2} J_{\sigma}(\mu) \\
J_{\sigma}(\mu) & =\int_{0}^{\pi / 2} d \theta \frac{\cos ^{\sigma+1} \theta}{\sqrt{1-\mu \sin ^{2} \theta}}
\end{aligned}
$$

and $K$ and $E$ are the complete elliptic integrals of the first and second kind respectively:

$$
\begin{aligned}
& K(\mu)=\int_{0}^{\pi / 2} \frac{d \theta}{\sqrt{1-\mu \sin ^{2} \theta}} \\
& E(\mu)=\int_{0}^{\pi / 2} d \theta \sqrt{1-\mu \sin ^{2} \theta}
\end{aligned}
$$




\section{Rutherford equation}

The Rutherford equation is obtained via the integration of the Maxwell-Ampère law

$$
-\nabla_{\perp}^{2} \tilde{\psi}=\mu_{0} \tilde{J}_{\|}
$$

Here we focus on the contribution of the perturbed ohmic current, due to the perturbed parallel electric field $\tilde{E}_{\|}$and perturbed plasma resistivity $\tilde{\eta}$, using the Spitzer resistivity dependence on temperature $\eta \propto T^{-3 / 2}:$

$$
\begin{aligned}
\tilde{J}_{\Omega} & =\tilde{E}_{\|} / \eta-J_{\Omega} \tilde{\eta} / \eta \\
& =\eta^{-1} \partial_{t} \psi_{1}\langle g\rangle+J_{\Omega} \frac{3}{2}\langle\tilde{T} / T\rangle
\end{aligned}
$$

with the flux surface average operator:

$$
\langle A\rangle=(\oint d \alpha A / \sqrt{\Omega+g}) /(\oint d \alpha / \sqrt{\Omega+g})
$$

Introducing the tearing stability index $\Delta^{\prime}$ Furth et al. (1963) and the normalized island width $W=w / a$, we obtain from the standard asymptotic matching procedure Rutherford (1973)

$$
I_{1} \tau_{R} \partial_{t} W=a \Delta^{\prime}+a \Delta_{\Omega}^{\prime}
$$

with $\tau_{R}=\mu_{0} a^{2} / \eta$ the resistive time and

$$
\begin{aligned}
I_{1} & =\sqrt{2} \int_{-1}^{\infty} d \Omega \oint \frac{d \alpha}{2 \pi} \frac{g\langle g\rangle}{\sqrt{\Omega+g}} \approx 0.82 \\
a \Delta_{\Omega}^{\prime} & =\frac{24}{W \sqrt{2}} \frac{q}{s} \frac{\mu_{0} R J_{\Omega}}{B_{z}} \int_{-1}^{\infty} d \Omega \oint \frac{d \alpha}{2 \pi} \frac{g\langle\tilde{T} / T\rangle}{\sqrt{\Omega+g}}
\end{aligned}
$$

We consider a large island (compared with the characteristic transport scale length Fitzpatrick (1995)), so that the temperature profile without RF heating is flat inside the island. The perturbed temperature is determined from $\langle\tilde{T} / T\rangle=(T(\Omega)-T(\Omega=1)) / T_{s}$, leading to

$$
\left\langle\frac{\tilde{T}}{\bar{T}}\right\rangle(\mu)=\frac{-T_{s}^{\prime}}{T_{s}} \frac{w}{4}\left(\frac{\pi}{4} \frac{P_{R F}^{t o t}}{P_{e q}}\right)^{1 / \sigma} F_{\sigma}\left(\mu, \mu_{c}\right)
$$

with

$$
\begin{aligned}
& F_{\sigma}\left(\mu<\mu_{c}, \mu_{c}\right)=\int_{\mu}^{\mu_{c}} \frac{d \mu^{\prime}}{\mu^{\prime 1 / 2}}\left\{\frac{1}{\mu^{\prime 1 / 2} J_{\sigma}\left(\mu^{\prime}\right)} \frac{E\left(\mu^{\prime}\right)+\left(\mu^{\prime}-1\right) K\left(\mu^{\prime}\right)}{E\left(\mu_{c}\right)+\left(\mu_{c}-1\right) K\left(\mu_{c}\right)}\right\}^{1 / \sigma}+\int_{\mu_{c}}^{1} \frac{d \mu^{\prime}}{\mu^{\prime 1 / 2}}\left\{\frac{1}{\mu^{\prime 1 / 2} J_{\sigma}\left(\mu^{\prime}\right)}\right\}^{1 / \sigma}(23) \\
& F_{\sigma}\left(\mu>\mu_{c}, \mu_{c}\right)=\int_{\mu}^{1} \frac{d \mu^{\prime}}{\mu^{\prime 1 / 2}}\left\{\frac{1}{\mu^{\prime 1 / 2} J_{\sigma}\left(\mu^{\prime}\right)}\right\}^{1 / \sigma}
\end{aligned}
$$

Note that the symmetry in $x$ of the temperature perturbation that is assumed here implies that the impact of the O-point heating on the bootstrap current perturbation Fitzpatrick (1995) is zero within this model.

The stabilizing contribution due to a localized heating can finally be expressed as

$$
\begin{aligned}
a \Delta_{\Omega}^{\prime} & =-\mathcal{F}_{\Omega}\left(\mu_{c}, \frac{P_{R F}^{t o t}}{P_{e q}}, \sigma\right) \frac{a}{\mathcal{J}} \frac{q}{s} \frac{\mu_{0} R J_{\Omega}}{B_{z}} \frac{P_{e q}}{N \chi_{\perp}^{0} T_{s}} \\
\mathcal{F}_{\Omega} & =\frac{3}{4 \pi^{2}} \mathcal{I}_{\Omega}\left(\mu_{c}, \sigma\right)\left(\frac{4}{\pi}\right)^{1-1 / \sigma}\left(\frac{P_{R F}^{t o t}}{P_{e q}}\right)^{1 / \sigma}
\end{aligned}
$$




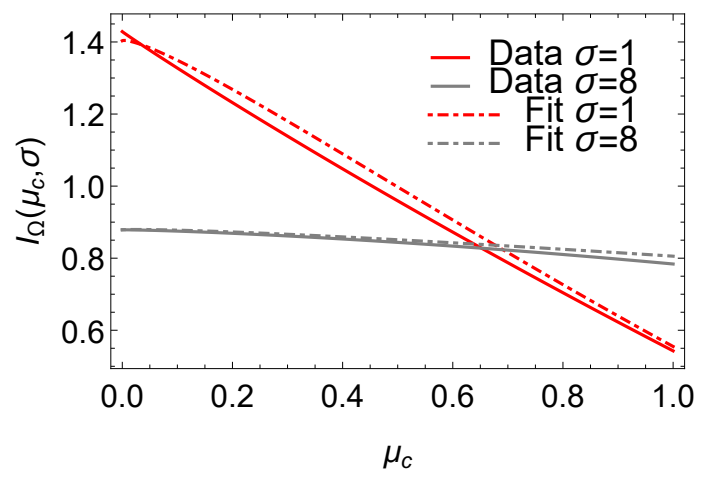

Figure 1: $\mathcal{I}_{\Omega}=f\left(\mu_{c}\right)$ and the fit given by equation 30 for $\sigma=1$ and $\sigma=8$.

with $\mathcal{I}_{\Omega}\left(\mu_{c}, \sigma\right)=\mathcal{I}_{1}\left(\mu_{c}, \sigma\right)+\mathcal{I}_{2}\left(\mu_{c}, \sigma\right)+\mathcal{I}_{3}\left(\mu_{c}, \sigma\right)$ and

$$
\begin{aligned}
& \mathcal{I}_{1}\left(\mu_{c}, \sigma\right)=\int_{0}^{\mu_{c}} d \mu[2 E(\mu)-K(\mu)] \int_{\mu}^{\mu_{c}} \frac{d \mu^{\prime}}{\mu^{\prime 1 / 2}}\left\{\frac{1}{\mu^{\prime 1 / 2} J_{\sigma}\left(\mu^{\prime}\right)} \frac{E\left(\mu^{\prime}\right)+\left(\mu^{\prime}-1\right) K\left(\mu^{\prime}\right)}{E\left(\mu_{c}\right)+\left(\mu_{c}-1\right) K\left(\mu_{c}\right)}\right\}^{1 / \sigma} \\
& \mathcal{I}_{2}\left(\mu_{c}, \sigma\right)=\left(\int_{0}^{\mu_{c}} d \mu[2 E(\mu)-K(\mu)]\right)\left(\int_{\mu_{c}}^{1} \frac{d \mu^{\prime}}{\mu^{\prime 1 / 2}}\left\{\frac{1}{\mu^{\prime 1 / 2} J_{\sigma}\left(\mu^{\prime}\right)}\right\}^{1 / \sigma}\right) \\
& \mathcal{I}_{3}\left(\mu_{c}, \sigma\right)=\int_{\mu_{c}}^{1} d \mu[2 E(\mu)-K(\mu)] \int_{\mu}^{1} \frac{d \mu^{\prime}}{\mu^{\prime 1 / 2}}\left\{\frac{1}{\mu^{\prime 1 / 2} J_{\sigma}\left(\mu^{\prime}\right)}\right\}^{1 / \sigma}
\end{aligned}
$$

The function $\mathcal{I}_{\Omega}$ is computed numerically and fitted with a simple formulae in the range $\left.\mu_{c} \in\right] 0,1[$ (39 points) and $\sigma \in[1,9]$ (9 points) with Mathematica Inc. (2016) (figure 1), so that the function $\mathcal{F}_{\Omega}$ can be approximated by:

$$
\mathcal{F}_{\Omega}\left(\mu_{c}, \frac{P_{R F}^{t o t}}{P_{e q}}, \sigma\right) \approx \frac{3}{4 \pi^{2}}\left[0.804+\frac{0.600}{\sigma}-1.091 \frac{\mu_{c}}{\sigma}+0.242\left(\frac{\mu_{c}}{\sigma}\right)^{2}-0.228 \frac{\mu_{c}}{\sigma} \ln \frac{\mu_{c}}{\sigma}\right]\left(\frac{P_{R F}^{t o t}}{P_{e q}}\right)^{1 / \sigma}
$$

and $\mathcal{F}_{\Omega}\left(\mu_{c}, \frac{P_{R F}^{t o t}}{P_{e q}}, \sigma \rightarrow \infty\right)$ tends to be a constant, independent of both $P_{R F}^{t o t}$ and $\mu_{c}$. For $\sigma=1$, the numerical evaluation of $\mathcal{F}_{\Omega}$ compares well with the results reported in Lazzari and Westerhof (2010), taking into account the modulation that is implicitely assumed in our model where the RF power is delivered only around the O-point. The time fraction where RF power is on is indeed $f^{\text {on }}=\pi^{-1} \arccos (1-$ $2 \mu_{c}$ ), and the effective stabilization is $a \Delta_{\Omega}^{\prime}(\sigma) \times f^{o n}$, except if a continuous O-point injection can be achieved Kasparek et al. (2016).

\section{Implications}

The importance of profile stiffness on the island stabilization capability by RF heating is illustrated by computing the quantity $a \Delta_{\Omega}^{\prime}(\sigma) \times f^{o n}$ as a function of $\mu_{c}$ and $P_{R F}^{t o t} / P_{e q}$ (figure 2), as well as the ratio $\Delta_{\Omega}^{\prime}(\sigma) / \Delta_{\Omega}^{\prime}(\sigma=1)$ (figure 3). We take as a typical value $\sigma=8$ expected in ITER Kinsey et al. (2011). For moderate values of $P_{R F}^{t o t} / P_{e q}$ (typically below unity), profile resilience is favourable since the heat diffusivity remains low inside the island as long as the temperature gradient stays below its equilibrium value, thus enhancing the effect of the RF power. For a typical medium size tokamak experiment (i.e. Asdex-Upgrade), taking $B=2.5 T, R=1.7 \mathrm{~m}, a=0.5 \mathrm{~m}, r=0.2 \mathrm{~m}, q=3 / 2, \mathrm{~s}=1, J_{\Omega}=5 \times 10^{5} \mathrm{~A} / \mathrm{m}^{2}$, $N=6 \times 10^{19} \mathrm{~m}^{-3}, T=2 \mathrm{keV}, \chi_{\perp}^{0}=2 \mathrm{~m}^{2} / \mathrm{s}, P_{R F}^{t o t}=2 \mathrm{MW}, P_{e q}=10 \mathrm{MW}$, and $\mu_{c}=0.25$, we have a $33 \%$ modulation scheme and an average stabilizing contribution from ohmic heating of $a \Delta_{\Omega}^{\prime}(\sigma) \times f^{o n} \approx-58$ for $\sigma=8$ instead of $(-20)$ for $\sigma=1$. The importance of the heating contribution to island stabilization with $\mathrm{RF}$ is therefore larger than computed so far, at least in the condition of perfect O-point heating, i.e. when power modulation is used. 


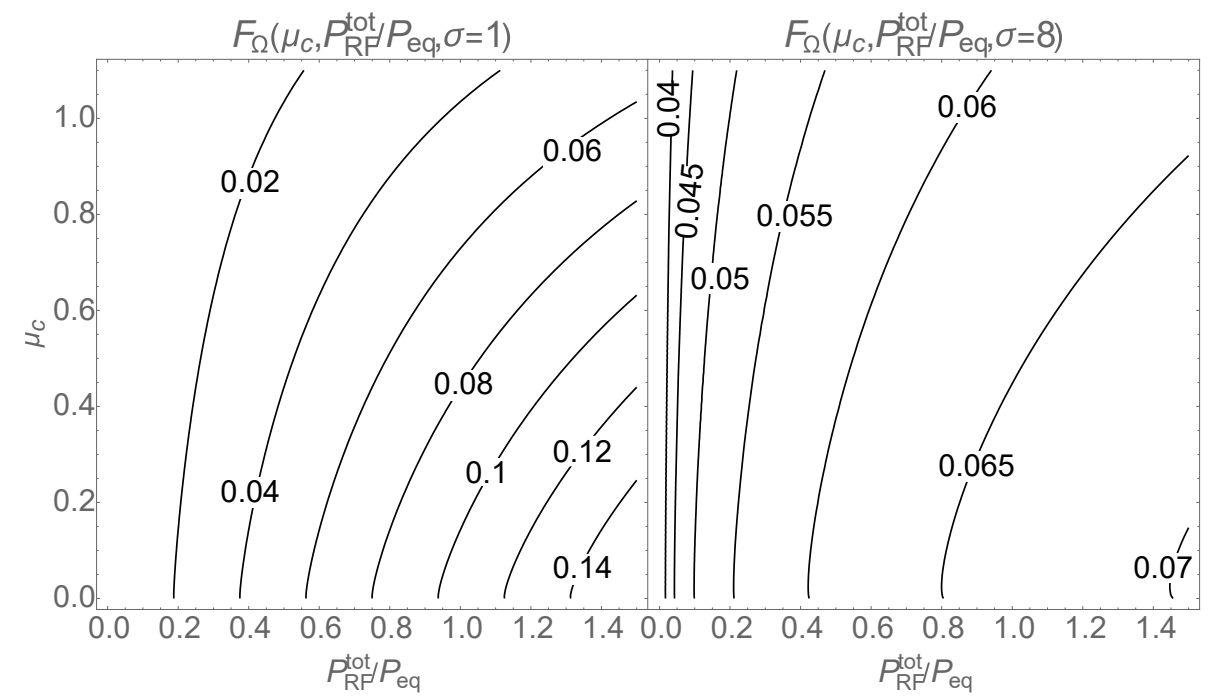

Figure 2: The function $\mathcal{F}_{\Omega}\left(\mu_{c}, \frac{P_{R F}^{t o t}}{P_{e q}}, \sigma\right)$ for $\sigma=1$ (left) and $\sigma=8$ (right).

\section{Acknowledgments}

This work has been carried out within the framework of the French Research Federation for Fusion Studies. It is part of the AMICI project funded by the Agence Nationale pour la Recherche (ANR-14CE32-0004-01).

\section{References}

Dimits, A. M., Bateman, G., Beer, M. A., Cohen, B. I., Dorland, W., Hammett, G. W., Kim, C., Kinsey, J. E., Kotschenreuther, M., Kritz, A. H., Lao, L. L., Mandrekas, J., Nevins, W. M., Parker, S. E., Redd, A. J., Shumaker, D. E., Sydora, R., and Weiland, J. (2000). Comparisons and physics basis of tokamak transport models and turbulence simulations. Physics of Plasmas, 7(3):969-983, DOI: 10.1063/1.873896, http://dx.doi.org/10.1063/1.873896.

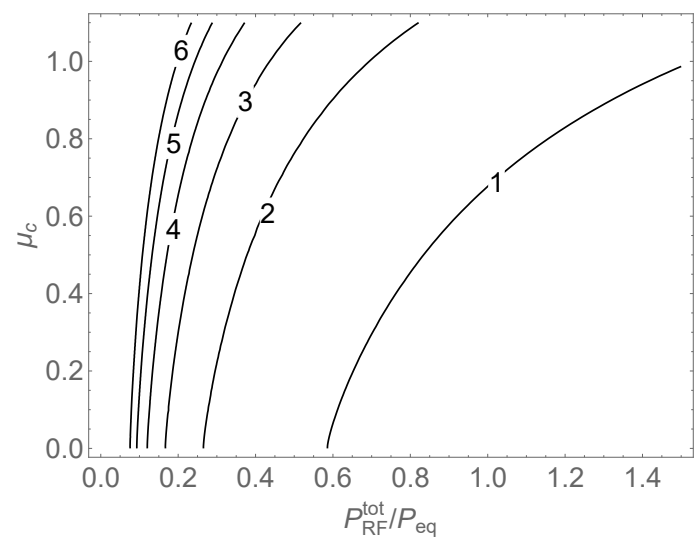

Figure 3: The ratio $\mathcal{F}_{\Omega}\left(\mu_{c}, \frac{P_{R F}^{t o t}}{P_{e q}}, \sigma=8\right) / \mathcal{F}_{\Omega}\left(\mu_{c}, \frac{P_{R F}^{t o t}}{P_{e q}}, \sigma=1\right)$. 
Fitzpatrick, R. (1995). Helical temperature perturbations associated with tearing modes in tokamak plasmas. Physics of Plasmas, 2(3):825-838, DOI: 10.1063/1.871434, http://link.aip.org/link/ ?PHP/2/825/1.

Fitzpatrick, R. (2017). Effect of nonlinear energy transport on neoclassical tearing mode stability in tokamak plasmas. Physics of Plasmas, 24(5):052504, DOI: 10.1063/1.4982610, http://dx.doi.org/ $10.1063 / 1.4982610$.

Furth, H. P., Killen, J., and Rosenbluth, M. (1963). Finite-resistivity instabilities of a sheet pinch. Physics of Fluids, 6(4):459-484.

Garbet, X., Mantica, P., Angioni, C., Asp, E., Baranov, Y., Bourdelle, C., Budny, R., Crisanti, F., Cordey, G., Garzotti, L., Kirneva, N., Hogeweij, D., Hoang, T., Imbeaux, F., Joffrin, E., Litaudon, X., Manini, A., McDonald, D. C., Nordman, H., Parail, V., Peeters, A., Ryter, F., Sozzi, C., Valovic, M., Tala, T., Thyagaraja, A., Voitsekhovitch, I., Weiland, J., Weisen, H., Zabolotsky, A., and the JET EFDA Contributors (2004). Physics of transport in tokamaks. Plasma Physics and Controlled Fusion, 46(12B):B557, http://stacks.iop.org/0741-3335/46/i=12B/a=045.

Hegna, C. C. and Callen, J. D. (1997). On the stabilization of neoclassical magnetohydrodynamic tearing modes using localized current drive or heating. Physics of Plasmas, 4(8):2940-2946, DOI: 10.1063/1.872426, http://link.aip.org/link/?PHP/4/2940/1.

Hornsby, W. A., Siccinio, M., Peeters, A. G., Poli, E., Snodin, A. P., Casson, F. J., Camenen, Y., and Szepesi, G. (2011). Interaction of turbulence with magnetic islands: effect on bootstrap current. Plasma Physics and Controlled Fusion, 53(5):054008, http://stacks.iop.org/0741-3335/53/i=5/ $\mathrm{a}=054008$.

Ida, K., Kamiya, K., Isayama, A., and Sakamoto, Y. (2012). Reduction of Ion Thermal Diffusivity Inside a Magnetic Island in JT-60U Tokamak Plasma. Phys. Rev. Lett., 109:065001, DOI: 10.1103/PhysRevLett.109.065001, https://link.aps.org/doi/10.1103/PhysRevLett.109. 065001.

Imbeaux, F., Ryter, F., and Garbet, X. (2001). Modelling of ech modulation experiments in asdex upgrade with an empirical critical temperature gradient length transport model. Plasma Physics and Controlled Fusion, 43(11):1503, http://stacks.iop.org/0741-3335/43/i=11/a=306.

Inagaki, S., Tamura, N., Ida, K., Nagayama, Y., Kawahata, K., Sudo, S., Morisaki, T., Tanaka, K., and Tokuzawa, T. (2004). Observation of Reduced Heat Transport inside the Magnetic Island O Point in the Large Helical Device. Phys. Rev. Lett., 92:055002, DOI: 10.1103/PhysRevLett.92.055002, https://link.aps.org/doi/10.1103/PhysRevLett.92.055002.

Inc., W. R. (2016). Mathematica, Version 11.0. Champaign, IL, 2016.

Kasparek, W., Doelman, N., Stober, J., Maraschek, M., Zohm, H., Monaco, F., Eixenberger, H., Klop, W., Wagner, D., Schubert, M., Schtz, H., Grnwald, G., Plaum, B., Munk, R., Schlter, K., and Team, A. U. (2016). NTM stabilization by alternating O-point EC current drive using a high-power diplexer. Nuclear Fusion, 56(12):126001, http://stacks.iop.org/0029-5515/56/i=12/a=126001.

Kinsey, J., Staebler, G., Candy, J., Waltz, R., and Budny, R. (2011). Iter predictions using the gyro verified and experimentally validated trapped gyro-landau fluid transport model. Nuclear Fusion, 51(8):083001, http://stacks . iop.org/0029-5515/51/i=8/a=083001.

Kurita, G., Tuda, T., Azumi, M., Takizuka, T., and Takeda, T. (1994). Effect of local heating on the $\mathrm{m}=2$ tearing mode in a tokamak. Nuclear Fusion, 34(11):1497, http://stacks.iop.org/0029-5515/ $34 / i=11 / a=I 08$.

Lazzari, D. D. and Westerhof, E. (2009). On the merits of heating and current drive for tearing mode stabilization. Nuclear Fusion, 49(7):075002, http://stacks. iop.org/0029-5515/49/i=7/a=075002. 
Lazzari, D. D. and Westerhof, E. (2010). On the merits of heating and current drive for tearing mode stabilization. Nuclear Fusion, 50(7):079801, http://stacks.iop.org/0029-5515/50/i=7/a=079801.

Rebut, P., Lallia, P., and Watkins, M. (1988). The critical temperature gradient model of plasma transport: applications to jet and future tokamaks. Plasma Physics and Controlled Nuclear Fusion Research, 2:191.

Rutherford, P. H. (1973). Nonlinear growth of the tearing mode. Physics of Fluids, 16(11):1903-1908, DOI: 10.1063/1.1694232, http://link.aip.org/link/?PFL/16/1903/1. 